\title{
Joint collision resolution and transmit-power adjustment for Aloha-type random access
}

\author{
Young-June Choi ${ }^{*}$ and Kang G. Shin ${ }^{2}$ \\ 1 Department of Computer Engineering, Ajou University, Suwon, Korea \\ 2 RTCL, Department of Electrical Engineering and Computer Science, University of Michigan, MI, U.S.A.
}

\begin{abstract}
We consider uplink random access for which slotted Aloha has usually been employed with unknown channel conditions. Upon failure of a transmission attempt, a user cannot tell whether the failure was caused by collision with other simultaneously transmitting users or by his use of insufficient transmit power. If a transmission attempt failed due to collision which could have been resolved by retransmission, increasing transmit power would just waste power and, moreover, reduce the other users' chance of successful access. To handle this lack of information on the cause of failure, we propose a novel Cause-of-Failure resolution, where the transmit power is increased after a given number of consecutive unsuccessful access attempts when the probability that a given failure is caused by collision becomes sufficiently low. To exploit the thusobtained transmit power for the next random access attempt, we also determine the Cause-of-Success based on the number of consecutive successful attempts, i.e., whether to (probabilistically) decrease or maintain the current transmit power. This way, users can adjust their transmit power for random access, which we call Auto Power Fallback (APF), considered as an advanced version of the power ramping algorithm. We evaluate APF by modeling analysis and numerical computation based on the slotted Aloha, showing that APF determines a suitable transmit power for uplink random accesses while achieving good performance. Copyright (C) 2011 John Wiley \& Sons, Ltd.
\end{abstract}

\section{KEYWORDS}

random access; slotted Aloha; power adjustment; auto power fallback

* Correspondence

Young-June Choi, Department of Computer Engineering, Ajou University, Suwon, Korea.

E-mail: choiyj@ajou.ac.kr

${ }^{\dagger}$ A 5-page summary of this work was presented at the INFOCOM'08 Mini-conference.

\section{INTRODUCTION}

The effective use of random access is important for uplink (i.e., from a user terminal to the base station) communications in a wireless network where the base station (BS) manages user terminals within its coverage area. Till date, Aloha-style random access mechanisms have been implemented mainly for uplink communications in cellular networks, and as its variant, exponential backoffs are used in certain systems, such as IEEE 802.11 systems. Current $2 \mathrm{G}$ and $3 \mathrm{G}$ wireless networks have deployed the slotted Aloha [1] for initial access, short message transmission, and various types of uplink signaling. In IEEE 802.16 wireless metropolitan area networks (WMANs), the use of random access is also extended to bandwidth requests and ranging [2-5].

In spite of its wide use, users usually do not know the proper level of transmit power to use for random access. It is practically impossible for a user to acquire the exact channel information for a successful transmission, and hence, a random access is attempted with only limited information of a channel before using it. In existing wireless systems, random access relies on this premise. Especially, random access is essential for network initialization when a new user enters the network. In some cases, users can estimate the condition of random access channel(s) by measuring the signal power of beacon or pilot (this assumption was used in opportunistic Aloha and channel-aware Aloha [5,6]). In OFDMA systems, however, this information might not be available because there are many channels suffering frequency-selective fading and pilots may not cover the whole subcarriers. Moreover, when the system is based on frequency division duplexing (FDD), the uplink channel has different characteristics from the downlink channel, thereby making it difficult to estimate the uplink channel condition from the downlink signals [3]. 
Devising an effective power-adjustment scheme is of great importance to such channel-condition-agnostic random access systems. A well-known problem in random access is the difficulty in distinguishing a collision from the failure caused by too low a transmit power. Increasing the transmit power aggressively (and/or blindly) to combat the failure will waste energy without any benefit. Hence, determining a suitable transmit power level to use is essential for energy savings, which is a critical issue in all wireless mobile systems.

To overcome the problem of power assignment, CDMA systems have already deployed a power-ramping algorithm [7]. In CDMA systems, code orthogonality that is critical for multiple access is exploited if all of the receive powers are identical, but this condition may not hold due to the near-far effect [8]. So, the power-ramping algorithm increases the transmit power by one step on failure of a random access attempt, while using the slotted Aloha. Following the algorithm in general random access systems, the transmit power would be increased even in case of collision-induced failures. It wastes energy by using excessive transmit power that also interferes with other users. Moreover, this algorithm becomes inefficient if the power ramping always starts from the initial transmit power level, although it can reuse the latest-used power level.

To remedy the above problems, we propose a random access solution that maintains a proper transmit power level. Unlike the conventional random access approach that relies on collision resolution using random backoffs, we develop a new concept of failure resolution that seeks an adequate transmit power level as well as collision resolution. When a random access attempt failed, the user did not know the cause of failure. We tackle this problem with a probabilistic approach and devise a Cause-of-Failure $(\mathrm{CoF})$ estimation algorithm that estimates whether the failure is caused by a collision or by use of insufficient transmit power.

Next, to reuse the thus-obtained transmit power level for the next random access attempt, we also propose a Cause-ofSuccess (CoS) estimation algorithm that estimates whether or not to decrease the current transmit power before attempting a random access. ${ }^{\dagger}$ This way, a random access need not start from the lowest transmit power level, thus ameliorating the network performance. The two algorithms determine whether to change the present power level according to the number of consecutive failures or successes. We call this mechanism Auto Power Fallback (APF), since the operation type is akin to the Auto Rate Fallback algorithm that adjusts the transmission rate to the link condition in IEEE 802.11 systems [9]. The auto rate fallback algorithm is not applicable to our system, because the BS is usually designed to be able to decode a basic data rate (i.e., given modulation and code rate) for the random access operation.

\footnotetext{
${ }^{\dagger}$ We develop this algorithm to examine the channel-condition-agnostic case. In some systems, an accurate power level may be tuned for the next access attempt following a successful access, but a success may not be guaranteed due to the unreliability of wireless channels.
}

In summary, we focus on uplink channels with the following features:

- Slotted Aloha is used for random access.

- The proper level of transmit power is not known.

- The basic data rate is only decodable for random access.

- The packet size of random access is fixed.

There have been numerous proposals for power control at the MAC layer, especially for IEEE 802.11 or ad hoc networks. Some of them (e.g., References [10,11]) deal with power control or energy consumption with respect to collisions within the transmission range for ad hoc networks. Their collision resolution relies on the MAC operation, so our approach should be compared to Aloha variants. In References [12-15], the transmit power was randomized, and especially, the authors of Reference [15] studied power control for slotted Aloha to improve system capacity under the assumption that power variations due to distance are eliminated by perfect power control. Also, transmit power is randomly given for CSMA random access to maximize network throughput in Reference [16].

The performance of Aloha under the near-far effect is addressed in Reference [8], and its stability studied in Reference [17] by adaptively varying the probability of retransmission. In Reference [18], a tree-splitting collision resolution protocol is proposed, which operates according to residual battery energy. The authors of Reference [19] studied a collision model for CDMA systems and referenced a number of power control methods. The authors of Reference [20] considered power control to meet the delay performance of slotted Aloha, assuming common feedback from the the access point. Power control with random access was also considered in References $[21,22]$ based on random backoffs rather than slotted Aloha, specifically for IEEE 802.16 systems. To the best of our knowledge, little has been done on resolving randomaccess failures by considering both collision resolution and transmit-power adjustment together for Aloha-type random access.

APF is close to the power-ramping algorithm and it can be referred to as an advanced power-ramping algorithm that employs failure resolution. While the power-ramping algorithm increases the transmit power aggressively, our algorithm focuses on power maintenance while considering both use of insufficient transmit power and collision as reasons for transmission failures. The power-ramping algorithm has many variants (see Reference [23] and references therein), but most of them have focused on the ramping size. Our algorithm can be applied to emerging systems such as the IEEE 802.16, as well as to ad hoc or mesh networks that require power-adjusted random access. However, we do not deal with IEEE 802.11 systems and the related ad hoc networks because they are based on a carrier-sensing protocol and thus need a power-control method that is compatible with the transmission range and link adaptation [10,11,24,25]. 
The performance of APF is evaluated based on the slotted Aloha in an environment that is affected primarily by path loss and shadowing. We also evaluate the successful access probability and energy consumption in steady-state, modeling it with a discrete-time Markov chain. Our primary objective is to study the transmit power management for fixed or nomadic terminals. When they move around, fast fading in addition to path loss makes the instantaneous channel condition fluctuate. It is well known that Rayleigh fading, considered as usual fast fading, will improve the performance of slotted Aloha [17,26]. So, allocating an appropriate transmit power may not be an important problem when random access channels are affected by fast fading due to the user's mobility. Our algorithm is also shown to perform well in those environments and provide useful insight in designing power-adjusted random access for a variety of wireless networks.

The remainder of this paper is organized as follows. Section 2 describes our system model and assumptions, and Section 3 proposes algorithms that consider both transmit power management and collision resolution together. Section 4 analyzes the performance of the algorithms by modeling it with a discrete-time Markov chain, and Section 5 evaluates them numerically and using simulation. We further discuss the applications of our approach and relevant implementation issues in Section 6. The paper concludes with Section 7.

\section{SYSTEM MODEL AND ASSUMPTIONS}

\subsection{Assumptions for slotted Aloha}

We consider a wireless network that is composed of multiple cells or clusters, each of which consists of a BS and many mobile users or terminals. As is commonly the case, random access is used for uplink communications from users to the BS. The air medium is assumed to be accessed randomly by following the slotted Aloha. A packet arriving (generated) during time slot $i$ is transmitted at time slot $i+1$. By the nature of random access, users are informed of the result of a transmission attempt only when it is successful. If the attempt was unsuccessful, there is usually no explicit response from the receiver, so users estimate the failure from the absence of ACK or the subsequent procedure (e.g., channel allocation) over the downlink. For the tractability of analysis, as in the literature (see, for example, Reference [4]), a user terminal is assumed to learn whether or not an access attempt was successful at the end of each slot from the BS's feedback.

Throughout this paper, we let $G$ denote the total effective offered load, under the assumption that random access

\footnotetext{
\# The access result might be fed back to the user within several slots (e.g., in 802.16 systems due to the decoding overhead) after the access attempt.
}

packets are of the same size as in bandwidth request. Since Aloha-type random access methods use random backoffs for their collision resolution, the total offered load includes new and backlogged packets. If a user's transmission attempt fails, he retransmits the same packet in a random backlogged manner. We assume an infinite number of users in the network, because the resultant performance is close to that of the case when the number of users is large as in typical wireless networks. Then, the traffic load generated in the network follows a Poisson distribution with mean rate $G$. Since a user's access attempt will succeed if the others do not attempt to access the network at the same time, the successful access probability, $P_{\text {succ }}$, of slotted Aloha is given by [4]

$$
P_{\text {succ }}=\exp (-G)
$$

The effective arrival rate $G$ may vary with time, but is usually averaged over a certain period of time. Moreover, since the number of users in a network usually does not change fast, we assume that a BS can measure the effective arrival rate which remains constant at $G$ for the time interval of interest.

\subsection{Channel condition and capture effect}

Since wireless channels are usually attenuated by path loss and fading, users experience different channel environments. Path loss PL is the most dominant factor in determining the channel condition for nomadic wireless networks (e.g., fixed 802.16). It is known to be a function of the distance $d$ between the transmitter and the receiver as follows [27]:

$$
\mathrm{PL}(\mathrm{dB})=A+10 \gamma \log _{10}\left(d / d_{\mathrm{o}}\right)+s, d \geq d_{\mathrm{o}},
$$

where $A$ and $s$ represent the path loss (in $\mathrm{dB}$ ) at distance $d_{\mathrm{o}}$ and the shadow fading variation, respectively, and $\gamma$ is the path loss exponent determined by urban or suburban characteristics.

Due to channel attenuation, the BS's receive powers from different users in a cell are not equal. Signal processing technology has enabled the BS to recognize a particular user's packet (capture effect) even when other users transmitted theirs at the same time, which would normally have been garbled up by collision. However, we do not consider the case where multiple users' random accesses are all successful $[6,28]$, since usually receivers are still designed for capturing one user's packet at a time to reduce the implementation overhead, which will trigger a simple subsequent operation like dedicating a channel or admitting a new user as a result of random access.

A certain user's random access attempt can be regarded as successful due to the capture effect even if other users attempted to access the network at the same time. That is, when the received power from a user $k$ is larger than the sum of other users' and background noise $\mathcal{N}$, which is equivalent 
to receive signal-to-interference-and-noise ratio (SINR), $\Gamma$, by a given threshold $\alpha$, user $k$ 's packet can be captured by the receiver. Throughout our analysis, we assume that the sum of noise and intercell interference does not vary, although intercell interference may cause fluctuation of SINR when a high frequency reuse factor such as 1 is used [4]. Our simulation-based evaluation in Section 5 reveals that, even when the same random access channel is used in neighboring cells for the frequency reuse factor of 1 , the impact of intercell interference is not significant in most cases due to the nature of random access and power adjustment of our scheme.

Let $\mathcal{R}_{k}$ be the BS's receive power of user $k$ 's transmission, then user $k$ 's packet will be captured if

$$
\Gamma_{k}=\frac{\mathcal{R}_{k}}{\sum_{i \neq k} \mathcal{R}_{i}+\mathcal{N}} \geq \alpha,
$$

where the threshold $\alpha$ is a design parameter and can be adjusted according to the code rate deployed at the transmitters $[13,15]$. How to set $\alpha$ is beyond the scope of this paper, and hence, will not be considered any further. We simply assume that $\alpha$ is set to be larger than the minimum SINR required for the BS to decode the transmitted data correctly. Typically, $\alpha$ is set to $5 \mathrm{~dB}$ or determined according to the standard of each wireless communication system.

\subsection{Power adjustment}

For power-adjusted random access, the BS is made to receive all packets with the same receive power. However, all the users in a cell are positioned at different locations and may be subject to different channel conditions. Each user should therefore adjust his transmit power so that the BS's receive power from the users' transmissions may lie in the same range. We obtain the minimal receive power $\mathcal{R}^{*}=\mathcal{N} \alpha$ from the definition of SINR, when there are no other simultaneously transmitting users. Therefore, we make each user adjust his transmit power autonomously to make the BS's receive power of his transmission not smaller than $\mathcal{R}^{*}$. Suppose there are $N$ transmit power levels, $\mathcal{T}^{1}, \cdots, \mathcal{T}^{N}\left(\mathcal{T}^{1}<\cdots<\mathcal{T}^{N}\right){ }^{\S}$ We assume that the BS can always decode a transmitted signal within a target bit error rate by any subset of $N$ levels. To meet $\mathcal{R}^{*}$, the transmit power can be increased in a way a priori agreed upon, as discussed in the next section.

The suitable transmit power level is referred to as the reference power level, which is used by most users, called reference-power users. However, some users, called excess-power users, will use more power than their own reference power, and some users, called deficient-power users, attempt to transmit at a lower level than their reference power level. If the reference power level is $n^{*}$, the excess-

\footnotetext{
$\S$ In this paper, we use $\mathcal{T}^{n}$ and $n$ interchangeably to represent a transmit power level.
}

power users transmit packets at levels $n^{*}+1, \cdots, N$. The users using $i$ levels higher power than their reference power are denoted by $i$-excess-power users. Note that the reference power level $n^{*}$ is user-specific, i.e., each user sets his own reference power level according to his location or channel condition. For analytical simplicity, we assume that access attempts of reference-power users do not cause collision to any excess-power users, and similarly, $i$-excess-power users do not cause collision to $(i+1)$-excess power users. These assumptions hold asymptotically when the step-size of transmit power levels gets large (e.g., 2-5 dB) as in random-access applications. They may not hold with a small probability when there are more than one simultaneously transmitting users of smaller $i$, but mostly hold as a result of the capture effect. We will later study this in detail via simulation.

\section{POWER-ADJUSTED RANDOM ACCESS}

\subsection{Problem formulation}

If every user maintains his transmit power such that the BS's receive power of his transmission is not smaller than $\mathcal{R}^{*}$, then a new user's initial access attempt may fail due to his collision with other users' attempts or use of insufficient transmit power. Collisions occur if there are other simultaneously transmitting users. We attribute the failure to use of insufficient transmit power if the new user's data is received at a power level lower than $\mathcal{R}^{*}$. Occasionally, a user's attempt with a proper transmit power may fail when other simultaneously transmitting users use excessive transmit power. We regard this case as collision, not the problem of using insufficient transmit power.

However, a user does not know whether the failure is caused by collision or insufficient transmit power, because the result of a random access attempt is acknowledged only when it was successful. If a user increases his transmit power aggressively to avoid low-transmit-power-induced failures, it may exceed the required receive power and waste energy. Hence, it is important for a user to decide whether to retry the failed transmission with the same or increased power level. To this end, we propose a new concept of failure resolution by extending the existing collision resolution as discussed next.

\subsection{Cause-of-Failure (CoF) estimation}

To prescribe a correct action after each transmission failure, we propose a probabilistic approach for determining the Cause-of-Failure $(\mathrm{CoF})$. First, we define $p_{\mathrm{c}}$ and $p_{1}$ to represent the probabilities of a random-access failure due to collision and use of insufficient transmit power, respectively. The basic idea is to estimate $p_{\mathrm{c}}$ and $p_{1}$ upon failure of a user's random access attempt. The failure caused by collision means that the transmit power used was sufficient. 
In other words, collision can occur only when the transmit power is not insufficient. So, we can decide on the $\mathrm{CoF}$ by comparing $p_{\mathrm{c}} \cdot\left(1-p_{1}\right)$ and a given threshold $\varepsilon$.

If $p_{\mathrm{c}} \cdot\left(1-p_{1}\right) \geq \varepsilon$, the user can retry the failed transmission with the same transmit power. Otherwise (i.e., $\left.p_{\mathrm{c}} \cdot\left(1-p_{\mathrm{l}}\right)<\varepsilon\right)$, the user can retry the transmission with more power, assuming that the failure was due to use of insufficient transmit power. The design parameter, $\varepsilon$, lies in 0,1 and determines the tendency of power increase. As $\varepsilon \rightarrow 0$, collision becomes a dominant $\mathrm{CoF}$, while insufficient transmit power becomes a dominant $\mathrm{CoF}$ as $\varepsilon \rightarrow 1$. Note that the case of $\varepsilon=1$ represents the conventional power-ramping algorithm; a transmission failure is always accompanied by a power increase at the next attempt.

Let $\Psi(n)$ be the probability that the reference transmit power level is $n$ within a cell. We simply let $\sum_{i=1}^{N} \Psi(i)=1$. According to user distribution in a cell, the BS can obtain $\Psi(n)$ for the coverage area that requires the transmit power level $\mathcal{T}^{n}$. This issue will be discussed further in Section 6 .

Let $m$ and $n(n=1, \cdots, N)$ be the number of consecutive failed transmissions and the index of transmit power level, respectively. We can then calculate $p_{\mathrm{c}}$ and $p_{1}$ as follows:"

$$
\begin{aligned}
& p_{\mathrm{c}}(m)=(1-\exp (-\widetilde{G}))^{m}, m \geq 1 \\
p_{1}(n)= & \operatorname{Pr}\left(\mathcal{R}<\mathcal{R}^{*} \mid \mathcal{T}^{n}\right) \\
= & \left(\sum_{i=n+1}^{N} \Psi(i), 1 \leq n \leq N-1,0, n=N\right.
\end{aligned}
$$

where $\widetilde{G}$ is the measured effective load, and $p_{\mathrm{c}}(m)$ is derived by the collision probability of slotted Aloha when the other users attempt to transmit at the same time. Clearly, $p_{\mathrm{c}}$ will decrease as the number of retransmissions increases and $p_{1}$ will decrease with the increase of power level.

Let $\phi_{n}$ be the number of consecutive transmission attempts with the power level $\mathcal{T}^{n}$ until the user increases his transmit power. Then,

$$
\phi_{n}=\operatorname{argmin}\left\{m: p_{\mathrm{c}}(m) \cdot\left(1-p_{\mathrm{l}}(n)\right)<\varepsilon\right\},
$$

where $\phi_{n}$ increases with the increase of $n$ as $p_{1}(n)$ decreases. Note that $p_{1}(N)=0$; in other words, failures are always caused by collisions at the highest transmit power under our assumption, but the number of transmission attempts is bounded by some maximum number for real applications. Usually, packets will be dropped after exhausting the maximum allowed number of attempts (using the final power level). Throughout this paper, we assume that failures can be recovered by an unlimited number of retransmissions at the maximum power level (i.e., $\phi_{N} \rightarrow \infty$ ) for analytical simplicity.

\footnotetext{
" In these parameters, we omit time and user indices for notational simplicity.
}

\subsection{Cause-of-Success (CoS) estimation}

When an initial random access was successful, the subsequent random access attempts can use the same transmit power instead of triggering the start of $\mathrm{CoF}$ algorithm from the lowest power level. If the channel condition remains unchanged, the user may reuse the latest-used (successful) power level for transmitting subsequent packets. However, the user's channel condition may change with time. Also, according to the $\mathrm{CoF}$ algorithm, with a slight probability, the power level may be increased even when successive collisions occur. If the present transmit power is too high for these reasons, causing the excessive receive power, it should be decreased. In our system model, however, the user does not know the channel condition for his next transmission. One possible way to handle this difficulty is to use the latestused power level, and from the result of using that power level, decide whether to change or retain the transmit power level.

We propose an algorithm to probabilistically determine the Cause-of-Success (CoS). While the $\mathrm{CoF}$ algorithm decides on the cause of failure after an unsuccessful random access, the CoS algorithm decides on the cause of success after making a successful random access. The CoS algorithm operates as follows. We define $p_{\mathrm{s}}$ and $p_{\mathrm{h}}$ as the probabilities of successful transmission without collision and with a power level causing the transmit power to be higher than the required level, respectively. If the success was due to collision-freedom, not high transmit power, the user can maintain his power level. Thus, if $p_{\mathrm{s}} \cdot\left(1-p_{\mathrm{h}}\right) \geq \delta$, the user retains the present power level for the next random access. Otherwise (i.e., $p_{\mathrm{s}} \cdot\left(1-p_{\mathrm{h}}\right)<\delta$ ), the user makes the next random access attempt with a decreased power level. $\delta$ is also a design parameter that determines the tendency of power decrease. Like $\varepsilon$, a user tends to decrease the present power level if $\delta \rightarrow 1$, and maintains it if $\delta \rightarrow 0$.

Let $m$ and $n$ be the number of consecutive successful transmissions and the index of present power level, respectively. We then obtain $p_{\mathrm{s}}$ and $p_{\mathrm{h}}$ as:

$$
\begin{gathered}
p_{\mathrm{s}}(m)=(\exp (-\widetilde{G}))^{m}, m \geq 1 \\
p_{\mathrm{h}}(n)=\left(1-p_{\mathrm{l}}(n-1)\right. \\
=\sum_{i=1}^{n-1} \Psi(i), 2 \leq n \leq N, 0, n=1
\end{gathered}
$$

where $p_{\mathrm{s}}(m)$ is derived by using the probability of no collision in slotted Aloha when no other users attempt to transmit.

Clearly, $p_{\mathrm{s}}(m)$ decreases with the increase of $m$. Hence, we calculate the number, $\varphi$, of consecutive successful transmissions until the user decreases his transmit power as

$$
\varphi_{n}=\operatorname{argmin}\left\{m: p_{\mathrm{s}}(m) \cdot\left(1-p_{\mathrm{h}}(n)\right)<\delta\right\} .
$$

Note that $\varphi_{1}$ is a trivial case since it already uses the lowest power level. 


\subsection{Auto power fallback}

We now describe how $\mathrm{CoF}$ and $\mathrm{CoS}$ algorithms operate in a real setting. Each user terminal can realize these algorithms in a distributed manner if users can measure, or be informed of, $\widetilde{G}$ and $\Psi(n)$ 's in order to calculate $p_{\mathrm{c}}, p_{\mathrm{l}}, p_{\mathrm{s}}$, and $p_{\mathrm{h}}$. Since this is impractical and/or inefficient, we develop instead a feasible mechanism for BSs to execute.

At each $\mathrm{BS}, p_{\mathrm{c}}$ and $p_{\mathrm{s}}$ are computed by estimating the traffic load $\widetilde{G}$ within its cell, and $p_{1}$ and $p_{\mathrm{h}}$ are computed by estimating $\Psi(n)$ 's from statistical users distribution and channel environment for given $N$ power levels. The BS then computes $\phi_{n}$ 's and $\varphi_{n}$ 's from Equations (6) and (9), respectively, and broadcasts the two sets every superframe via a common control channel or a beacon. Then, each user is informed of the number of consecutive successes or failures allowed until he decreases or increases his transmit power at each level. Note that $\phi_{n}$ 's and $\varphi_{n}$ 's are common to all the users in a cell.

The CoF and CoS algorithms fulfilled by each user are summarized below.

1: if initial access then

2: $\quad n=1, x=0, y=0$

3: end if

4: random access with transmit power level $n$

\section{5: if successful then}

6: $\quad x \leftarrow x+1, y=0$

7: $\quad$ if $x=\varphi_{n}$ then

8: $\quad n \leftarrow \max (n-1,1)$

9: $\quad x=0$

10: $\quad$ end if

11: else

12: $y \leftarrow y+1, x=0$

13: $\quad$ if $y=\phi_{n}$ then

14: $\quad n \leftarrow \min (n+1, N)$

15: $\quad y=0$

16: end if

17: $\quad$ arrange the next random access

\section{8: end if}

For example, when $N=10, G=0.75, \varepsilon=\delta=0.1$, and $\Psi(n)$ 's are used from Table I at $\gamma=4.0$ and $R=3 \mathrm{~km}$. The set of $\phi_{n}$ 's is given as $\{1,2,2,3,3,3,4,4,4,4\}$, and the set of $\varphi_{n} \mathrm{~s}$ as $\{4,3,3,3,3,3,2,2,2,2\}$. If a user's suitable power level is 5 , he first tries to transmit at levels $1,2,3$, and 4 , each with $\phi_{n}$ of $1,2,2$, and 3, respectively. After this number of failures, he will attempt a transmission at level 5. This example is depicted in Figure 1. The first failure at level 5 appears to have resulted from collision, and he finally succeeds with his second transmission attempt. After the success, he will use power level 5 for the next random access attempt. After 3 consecutive successes later at this level, he will decrease his transmit power according to the $\mathrm{CoS}$ algorithm. At level 4, as he suffered three consecutive failures, he increases the transmit power again according to the CoF algorithm.

This operation is akin to the auto rate fallback that is used for link adaptation in the IEEE 802.11 [9], where the transmission rate is increased or decreased when the user receives 10 consecutive ACKs (i.e., successes) or experiences 2 consecutive failures. Our mechanism replaces the link rate with the transmission power, and is adaptive to the power level, and called auto power fallback (APF). Like in the auto rate fallback, APF can fix $\phi$ and $\varphi$ by assigning $\varepsilon\left(1-p_{1}\right)$ and $\delta\left(1-p_{\mathrm{h}}\right)$ instead of $\varepsilon$ and $\delta$, respectively, thereby making the number of successive attempts irrelevant to the power level. This special case yields absolute fairness with respect to the successful access probability, and facilitates the implementation of our algorithm as there is no need to estimate $\Psi(n)$ s.

\section{PERFormance ANALYSIS}

APF drives every user to probabilistically and autonomously adjust his own transmit power to a suitable level. According to our definition in Section 2.3, those using a suitable transmit power level are called reference-power users. The traffic load of reference-power users is denoted by $G^{(0)}$. By the probabilistic nature of the APF algorithm, some users called excess-power users will use more power than their reference power, because users increase their transmit power after making $\phi_{n}$ unsuccessful transmission attempts even if the transmission failures had really been caused by collisions. We denote the traffic load of $i$-excess-power users by $G^{(i)}$. On the other hand, some users called deficient-power users attempt to transmit at a lower level than their reference power level $n^{*}$ due to the $\mathrm{CoS}$ algorithm. We denote their traffic load by $G^{(-1)}$.

Frequently-used symbols in our performance analysis are listed as follows:

- $G$ : total traffic load.

- $G^{(i)}$ : traffic load of reference-power users $(i=0)$, deficient-power users $(i=-1)$, or $i$-excessive-power users.

- $p_{\mathrm{c}}^{(i)}(m)$ : probability of $m$ consecutive random-access failures due to collision at reference-power level $(i=0)$, deficient-power level $(i=-1)$, or $i$-excessivepower levels. 
Table I. Transmit power levels for our simulation.

\begin{tabular}{|c|c|c|c|c|c|c|c|c|c|c|}
\hline$n$ & 1 & 2 & 3 & 4 & 5 & 6 & 7 & 8 & 9 & 10 \\
\hline $\mathcal{T}^{n}(\mathrm{dBm})$ & -25 & -20 & -15 & -10 & -5 & 0 & 5 & 10 & 15 & 20 \\
\hline$\Psi(\%)$ & 2.0 & 1.7 & 3.3 & 6.3 & 12.2 & 23.2 & 33.8 & 16.3 & 1.1 & 0.1 \\
\hline
\end{tabular}

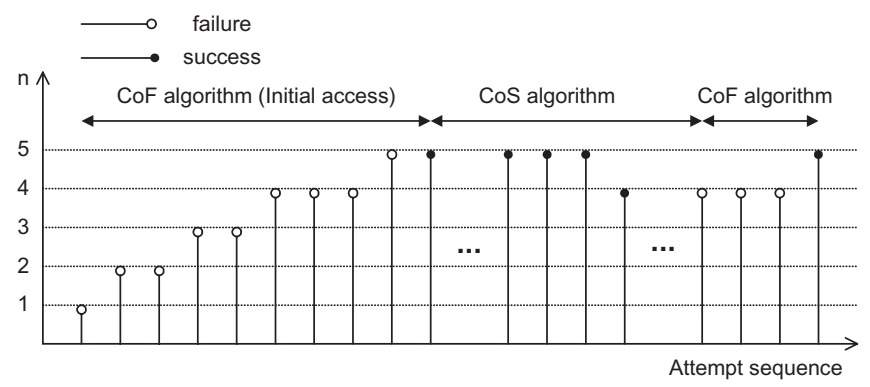

Figure 1. An example of auto power fallback mechanism.

- $p_{\mathrm{s}}^{(i)}(m)$ : probability of $m$ consecutive random-access successes due to collision-freedom at reference-power level $(i=0)$, deficient-power level $(i=-1)$, or $i$ excessive-power levels.

- $\alpha$ : minimum SINR required for the BS to decode the transmitted data correctly.

- $\Psi(n)$ : probability that the reference transmit power level is $n$ within a cell.

- $N$ : total number of transmit power levels.

- $\phi_{n}$ : number of consecutive transmission attempts at transmit power level $n$ until the power level is increased.

- $\varphi_{n}$ : number of consecutive transmission attempts at transmit power level $n$ until the power level is decreased.

Notice that the above parameters are cell-specific, but $n^{*}$ is user-specific.

According to the assumption in Section 2.3, $G^{(i)}$ dominates the others with the indices smaller than $i$ by the capture effect. In contrast, the users of $G^{(i)}$ cause collisions to the others with the indices not larger than $i$ for $i=0, \cdots, N-1$. Then, $p_{\mathrm{s}}^{(i)}\left(\varphi_{n^{*}+i}\right)$ and $p_{\mathrm{c}}^{(i)}\left(\phi_{n^{*}+i}\right)$, representing the $i$-excesspower users' probabilities of successes and collisions with the reference power level $n^{*}$ are given, respectively, by

$p_{\mathrm{s}}^{(i)}\left(\varphi_{n^{*}+i}\right)=\left[\exp \left(-\sum_{j=i}^{N} G^{(j)}\right)\right]^{\varphi_{n^{*}+i}}, 1 \leq i \leq N-n^{*}$

$$
\begin{aligned}
p_{\mathrm{c}}^{(i)}\left(\phi_{n *+i}\right) & =\left[1-\exp \left(-\sum_{j=i}^{N} G^{(j)}\right)\right]^{\phi_{n^{*}+i}}, \\
1 & \leq i \leq N-n^{*}-1 .
\end{aligned}
$$

Meanwhile, access attempts by deficient-power users can cause collision to any reference-power users by the definition of $\mathcal{R}^{*}$, so the probabilities of successes and collisions of reference-power users, $p_{\mathrm{s}}^{(0)}\left(\varphi_{n *}\right)$ and $p_{\mathrm{c}}^{(0)}\left(\phi_{n *}\right)$, are given by

$$
\begin{gathered}
p_{\mathrm{s}}^{(0)}\left(\varphi_{n^{*}}\right)=[\exp (-G)]^{\varphi_{n^{*}}}, \\
p_{\mathrm{c}}^{(0)}\left(\phi_{n^{*}}\right)=[1-\exp (-G)]^{\phi_{n^{*}}} .
\end{gathered}
$$

Using these probabilities, we can build a discrete-time Markov chain where each state represents a transmit power level [29]. Let $P_{n *}(i+1 \mid i)$ represent the probability of transitioning from state $i$ to state $i+1$ when the reference level is $n^{*}$, then the state transition probabilities are

$$
\begin{gathered}
P_{n^{*}}(i+1 \mid i)=\left(\begin{array}{c}
1,1 \leq i \leq n^{*}-1 \\
p_{\mathrm{c}}^{\left(i-n^{*}\right)}\left(\phi_{i}\right), n^{*} \leq i \leq N-1
\end{array}\right. \\
P_{n^{*}}(i-1 \mid i)=\left(\begin{array}{c}
0,2 \leq i \leq n^{*}-1 \\
p_{\mathrm{s}}^{\left(i-n^{*}\right)}\left(\varphi_{i}\right), n^{*} \leq i \leq N .
\end{array}\right.
\end{gathered}
$$

We define $\Pi_{n^{*}}(-1), \Pi_{n^{*}}(0), \Pi_{n^{*}}(1), \cdots, \Pi_{n^{*}}\left(N-n^{*}\right)$ as the probabilities that a user's power level becomes $n^{*}-1, n^{*}, n^{*}+1, \cdots, N$ in steady-state. As $\sum_{i=-1}^{N-n^{*}} \Pi_{n^{*}}(i)=1$ for any $n^{*}$, for $i=-1,0, \cdots, N-n^{*}$, we obtain $\Pi_{n^{*}}(i)$ that is expressed by transition probabilities.

The traffic load by deficient-power users is expressed by

$$
G^{(-1)}=G \cdot \sum_{i=2}^{N} \Psi(i) \cdot \Pi_{i}(-1) .
$$

Similarly, the traffic loads by reference-power users and excess-power users are given by

$$
G^{(0)}=G \cdot \sum_{i=1}^{N} \Psi(i) \cdot \Pi_{i}(0),
$$




$$
G^{(1)}=G \cdot \sum_{i=1}^{N-1} \Psi(i) \cdot \Pi_{i}(1),
$$

$$
G^{(N-1)}=G \cdot \Psi(1) \cdot \Pi_{1}(N-1),
$$

where $\sum_{i=-1}^{N-1} G^{(i)}=G$.

As $G^{(i)}$ is a function of $p_{\mathrm{s}} \mathrm{s}$ and $p_{\mathrm{c}} \mathrm{s}$, and each $p_{\mathrm{s}}$ and $p_{\mathrm{c}}$ are derived from $G^{(i)} \mathrm{s}$, it is impossible to calculate them directly, but we can solve it recursively by setting $G$ to the initial value of $G^{(0)}$ and 0 to the others.

From $G^{(i)}$ s, we obtain the successful access probability in a cell, $\bar{P}_{\text {succ }}$, as:

$$
\bar{P}_{\text {succ }}=\frac{G^{(0)}}{G} \exp (-G)+\sum_{i=1}^{N-1} \frac{G^{(i)}}{G} \exp \left(-\sum_{j=i}^{N-1} G^{(j)}\right) .
$$

Let $P_{\text {succ }}\left(n^{*}\right)$ be the successful access probability of users with reference level $n^{*}$, which is given by

$$
\begin{aligned}
P_{\text {succ }}\left(n^{*}\right)= & \Pi_{n^{*}}(0) \exp (-G) \\
& \sum_{i=1}^{N-n^{*}} \Pi_{n^{*}}(i) \exp \left(-\sum_{j=i}^{N-1} G^{(j)}\right) .
\end{aligned}
$$

We now examine the average number of transmission attempts, $\bar{\chi}_{n^{*}, S}$, and the average energy consumption in steady-state, $\overline{\mathcal{E}}_{n^{*}, S}$, for making random access attempts until it succeeds when the reference power level is $n^{*}$. Let $\bar{\chi}_{n^{*}}^{(i)}$ indicate the average number of transmission attempts at $i$ excess-power level when the reference level is $n^{*}$, and the case of $i=0$ represents the average number of transmission attempts at the reference level. If the transmission is successful at the $j$ th attempt with a certain power level, then there must have been $(j-1)$ collisions with this power level. Then, $\bar{\chi}_{n^{*}}^{(i)}$ is expressed by

$$
\bar{\chi}_{n^{*}}^{(i)}=\sum_{j=1}^{\phi_{n^{*}+i}} j \cdot p_{\mathrm{c}}^{(i)}(j-1) \cdot\left(1-p_{\mathrm{c}}^{(i)}(1)\right),\left(i=0, \cdots, N-n^{*}\right) .
$$

Let $\mathcal{W}_{i}$ be the energy consumed for transmitting a random access packet with transmit power level $i$, then we obtain $\overline{\mathcal{E}}_{n^{*}, S}$ as:

$$
\begin{aligned}
\overline{\mathcal{E}}_{n^{*}, S}= & \Pi_{n^{*}-1}\left(\phi_{n^{*}-1} \mathcal{W}_{n^{*}-1}\right. \\
& \left.+\sum_{i=0}^{N-n^{*}} \bar{\chi}_{n^{*}}^{(i)} \mathcal{W}_{n^{*}+i} \prod_{j=n^{*}}^{n^{*}+i-1} p_{\mathrm{c}}^{\left(j-n^{*}\right)}\left(\phi_{j}\right)\right)
\end{aligned}
$$

$$
\begin{array}{r}
+\Pi_{n^{*}}\left(\sum_{i=0}^{N-n^{*}} \bar{\chi}_{n^{*}}^{(i)} \mathcal{W}_{n^{*}+i} \prod_{j=n^{*}}^{n^{*}+i-1} p_{\mathrm{c}}^{\left(j-n^{*}\right)}\left(\phi_{j}\right)\right) \\
+\Pi_{n^{*}+1}\left(\sum_{i=1}^{N-n^{*}} \bar{\chi}_{n^{*}}^{(i)} \mathcal{W}_{n^{*}+i} \prod_{j=n^{*}+1}^{n^{*}+i-1} p_{\mathrm{c}}^{\left(j-n^{*}\right)}\left(\phi_{j}\right)\right) \\
+\cdots+\Pi_{N} \bar{\chi}_{n^{*}}^{\left(N-n^{*}\right)} \mathcal{W}_{N},
\end{array}
$$

where we let $\prod_{j=n^{*}}^{n^{*}-1} p_{\mathrm{c}}^{\left(j-n^{*}\right)}\left(\phi_{j}\right)=\prod_{j=n^{*}+1}^{n^{*}} p_{\mathrm{c}}^{\left(j-n^{*}\right)}\left(\phi_{j}\right)=1$ for notational simplicity. We omit the expression of $\bar{\chi}_{n^{*}, S}$ since it is an extraction of all $\mathcal{W}_{i}$ s from Equation (23).

Finally, we derive the average number of transmission attempts, $\bar{\chi}_{n^{*}, I}$, and the average amount of energy consumption, $\overline{\mathcal{E}}_{n^{*}, I}$, required to succeed in the initial access when the reference level is $n^{*}$. Usually, the initial access begins from the lowest power level, $n=1$, since the channel condition is not known. If a user's reference power is $n^{*}$, his random access is most likely made with $\mathcal{T}^{n^{*}}$. That is, the random access cannot be successful when the power level is lower than $n^{*}$. Hence, we have

$$
\bar{\chi}_{n^{*}, I}=\left(\sum_{i=1}^{n^{*}-1} \phi_{i}\right)+\bar{\chi}_{n^{*}}^{(0)}+\sum_{i=1}^{N-n^{*}} \bar{\chi}_{n^{*}}^{(i)} \prod_{j=n^{*}}^{n^{*}+i-1} p_{\mathrm{c}}^{\left(j-n^{*}\right)}\left(\phi_{j}\right)
$$

where $\sum_{i=1}^{n^{*}-1} \phi_{i}=0$ for $n^{*}=1$. In Equation (24), the last term implies the number of transmission attempts at excessive transmit power $\mathcal{T}^{i}\left(i=n^{*}+1, \cdots, N\right)$. Similarly, $\overline{\mathcal{E}}_{n^{*}, I}$ is given by

$$
\begin{aligned}
\overline{\mathcal{E}}_{n^{*}, I}= & \left(\sum_{i=1}^{n^{*}-1} \phi_{i} \mathcal{W}_{i}\right)+\bar{\chi}_{n^{*}}^{(0)} \mathcal{W}_{n^{*}} \\
& +\sum_{i=1}^{N-n^{*}} \bar{\chi}_{n^{*}}^{(i)} \mathcal{W}_{n^{*}+i} \prod_{j=n^{*}}^{n^{*}+i-1} p_{\mathrm{c}}^{\left(j-n^{*}\right)}\left(\phi_{j}\right) .
\end{aligned}
$$

\section{NUMERICAL RESULTS}

\subsection{Evaluation environment}

To evaluate the performance of APF, we consider a single cell with a radius of $1 \mathrm{~km}$. We will consider the intercell interference in a multicell environment after presenting the performance results in a single cell environment. We emulated the IEEE 802.16 systems for various parameters and uplink packet generations [2]. For uniformly distributed users within a cell, we compute the probability density function (pdf) of transmit power required to meet the requirement of $\alpha=5 \mathrm{~dB}$. Table I shows the computed $\Psi$ s according to the power levels, when the transmit 
power is increased or decreased by a constant amount of $5 \mathrm{~dB}$ in case of $N=10$. This table is obtained for the background noise of $-96 \mathrm{dBm}, \gamma$ (path-loss exponent) of 3.5, and the average channel condition; that is, the shadowing effect is averaged out. Each cell exhibits different channel characteristics, depending on whether it is an urban or suburban type [30], so it can estimate $\Psi(n)$ from its overall user distribution and channel model.

To verify our analysis results, we conducted simulation, and obtained the averaged performance of 1000000 events. In each event, 100 users are randomly distributed in a cell without changing the position, while generating packets with Poisson inter-arrivals. Random access packets of the same size are generated for bandwidth requests. In the slotted Aloha, when a random access failed, it is backlogged and retransmitted after a random amount of time elapses, but we set a maximum of 100 slots to prevent infinite backlogs. We consider the bandwidth of $500 \mathrm{kHz}$ for a random access channel, assuming that $5 \%$ of total bandwidth $(10 \mathrm{MHz})$ is used for random access [2]. The Gaussian shadowing has zero mean and variance of $8 \mathrm{~dB}$ [31].

By observing the number of random accesses ${ }^{\text {II }}$ in the cell, the BS in the simulator estimates $G$ to be one of $0.25,0.5$, $0.75,1$, and 1.25 . Note that accurate estimation of $G$ is not needed, because $\phi_{n} \mathrm{~s}$ and $\varphi$ s are changed slightly even when $G$ is increased by 0.25 . We confirmed via simulation that the performance is almost insensitive to the change of estimated $G$ and we omit the result in this paper.

We use a default value of $\alpha=5 \mathrm{~dB}$. Since we use $5 \mathrm{~dB}$ as the difference between any two transmit-power levels in Table I, the simulation result of $\alpha=5 \mathrm{~dB}$ is closest to our analysis, which will be confirmed next.

\subsection{Comparison with other schemes}

To demonstrate the APF's performance superiority, we compare it with other schemes: full-power, random-power, and power-ramping schemes. In the full-power scheme, random access is always attempted with full power $(20 \mathrm{dBm}$ in our simulation). In the random-power scheme, a transmit power is randomly chosen among the 10 values given in Table I. The power-ramping scheme is a special case of APF (i.e., $\varepsilon=1$ ). Therefore, the power-ramping algorithm is shown to perform sometimes as good as APF.

\subsubsection{Comparison of reference power ratio.}

The objective of APF is to find a reference power level for random access. To show how well the proposed scheme tunes each user's transmit power to a reference power level, we introduce a Reference Power Ratio (RPR) that is derived from the ratio of reference-power users. The RPR is easily

II Even if a collision occurs in practice, the BS can detect it owing to recent signal processing technologies.

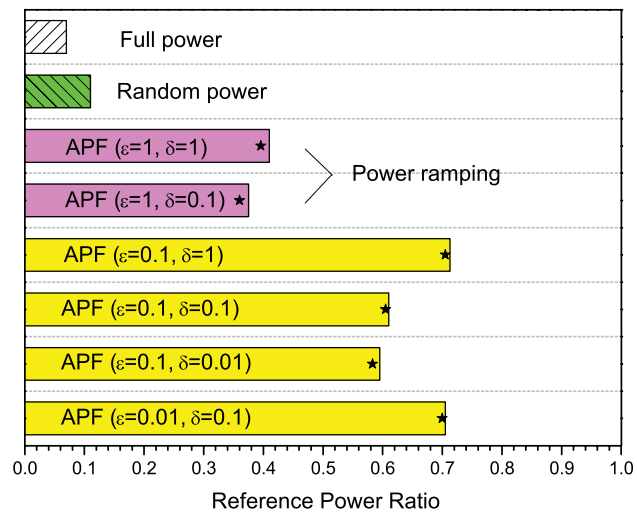

Figure 2. Comparison of the reference-power ratio among APF and other schemes ( $G=0.75$ in steady-state). Bars represent simulation results and symbols represent analysis results.

computed as

$$
\mathrm{RPR}=G^{(0)} / G
$$

Thus, we compare APF with other schemes in terms of RPR, consumed energy, and throughput.

Figure 2 compares the RPR of APF and other schemes. The full-power and random-power schemes show very small RPRs, because they do not consider power adjustment. The power-ramping scheme can also be represented as $\mathrm{APF}$ when $\varepsilon=1$. In this case, the transmit power is aggressively increased in case of failure, so the RPR is relatively small compared to other APF cases. Hence, in general, APF satisfies higher RPRs than existing schemes.

To validate our analysis, we compared the RPR obtained from the analysis with its corresponding simulation result. While our analysis assumed that $G^{(i)}$ does not cause collision to the others with the index larger than $i$ for $i=0, \cdots, N-2$ in Equations (10) and (11), this does not hold in the simulation result as in a real case, when there are more than one user of the same $G^{(i)}$. As shown in the result, however, it does not affect the performance significantly because of its low probability. As the capture effect is more pronounced in the simulation from diverse receive powers, its RPR is higher than the analysis result. From now on, we will display only the simulation results for readability and also because they show a similar tendency to the analytic results.

\subsubsection{Comparison of consumed energy.}

To calculate the amount of energy consumption without considering the effect of any other factor, we do not consider the energy consumed by other processing, and assume that the transmission duration for a random access packet is fixed at $2 \mathrm{~ms}$.

Figure 3 compares the average energy consumption of APF and other schemes when $G=0.75$. APF consumes much less energy than the full-power scheme, even though APF transits to different states. Surprisingly, APF consumes only $15-25 \%$ of the energy consumed by the full transmit 


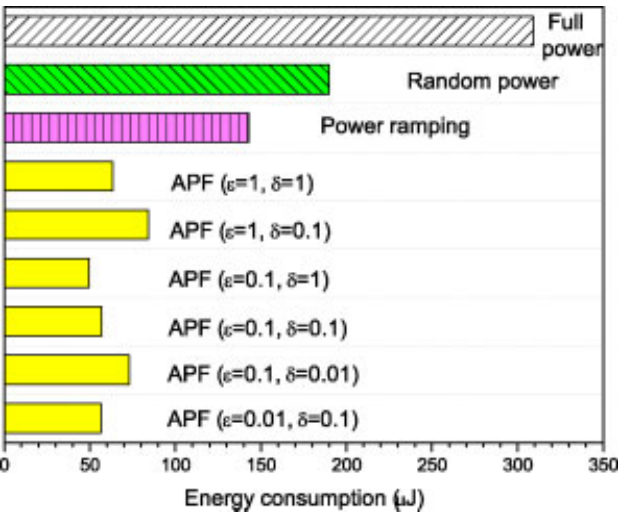

Figure 3. Comparison of the average energy consumption among APF and other schemes ( $G=0.75$ in steady-state).

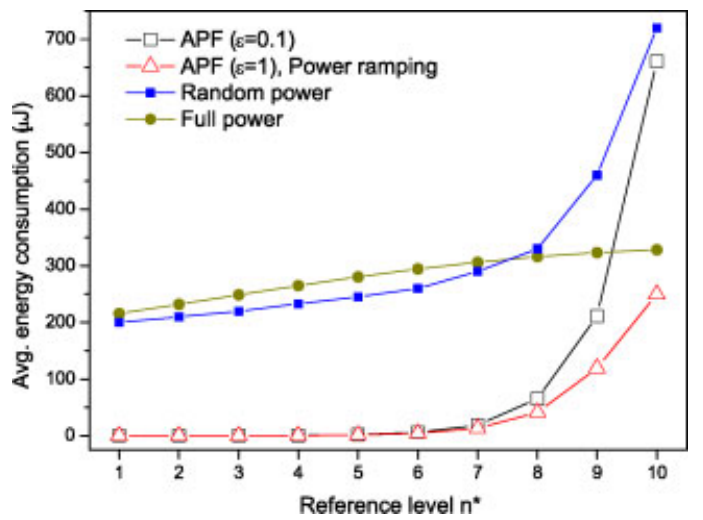

Figure 4. Comparison of energy consumption for initial access $(G=0.75)$.

power scheme. We also obtained the consumed energy by the power-ramping scheme without applying our $\mathrm{CoS}$ algorithm. Then, its consumed energy is higher than APF, as more transmissions are needed for each random access.

The case of $\varepsilon=1$ in APF is close to the pattern of power ramping. We observe that the case of $\varepsilon=1$ is not beneficial for the energy savings because it does not distinguish a collision from use of an insufficient power level. The case of $\delta=1$ is the reversed pattern of power ramping in that the transmit power is reduced on each successful transmission. Thus, as in the case $\varepsilon=0.1$ and $\delta=1$, the aggressive power decrease yields a small amount of energy consumption.

Figure 4 plots the energy consumption of initial access. At all but the final power level, APF can leverage the energy savings during the initial access, unlike the case of fullpower transmission, although several transmission attempts might be necessary at each level to succeed. The amount of energy consumption by power ramping is equivalent to the case of $\varepsilon=1$ in APF and shows more efficient than $\varepsilon=0.1$, because it requires only one transmission attempt at each level, and thus it has been designed for initial access.

\subsubsection{Comparison of throughput.}

Figure 5 compares the throughput of full power, random power, and APF schemes at $G=0.75$. We found that the

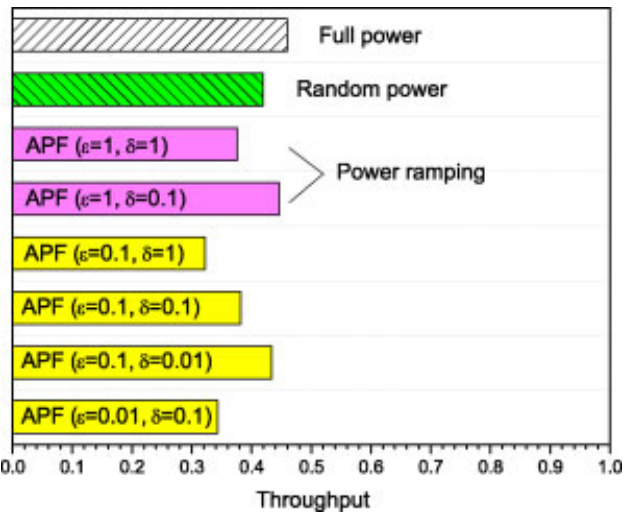

Figure 5. Comparison of throughput ( $G=0.75$ in steady-state).

curve for $\varepsilon=0.01$ and $\delta=0.1$ of $\mathrm{APF}$ is closest to the well-known throughput of slotted Aloha, as the ratio of excessive-power users is small. Other APF cases mostly show better throughput by virtue of the capture effect from excessive-power users whose ratio is relatively higher. The case of $\varepsilon=1$ is equivalent to power ramping, and this aggressive power increase contributes to the throughput while it does not to energy consumption. Conversely, the aggressive power decrease, shown at $\delta=1$, contributes to the energy consumption, but not to the throughput. Hence, there is a tradeoff between the energy savings and the throughput.

The full-power and random-power schemes show relatively high throughput but at the cost of much larger energy consumption. Although high throughput is achieved by the full-power scheme, this scheme is not desirable for uplink transmissions, because every random access uses full transmit power, quickly draining energy from wireless user devices.

Table II summarizes the effects of several $\varepsilon s$ and $\delta$ s on RPR, energy savings, throughput, and initial access, where ' $O$ ' and ' $X$ ' mean their positive and negative impacts, respectively, and ' $\Delta$ ' means relative neutrality. As stated earlier, there is a tradeoff between energy savings and throughput. This trend found in $\varepsilon$ holds conversely in $\delta$. Depending on the policy of energy-sensitivity or performance-efficiency, the system can choose these parameters, and also dynamically change them according to the user condition.

\subsection{Other considerations}

We now present the effect of $\alpha$, fast fading, and intercell interference on APF.

\subsubsection{Effect of $\alpha$.}

To investigate the effect of $\alpha$, we define an excess-power ratio (EPR) as

$$
\mathrm{EPR}=1-\left(G^{(-1)}+G^{(0)}\right) / G .
$$


Table II. Summary of impacts of selecting $\varepsilon$ and $\delta$.

\begin{tabular}{|c|c|c|c|c|c|c|}
\hline & \multicolumn{3}{|c|}{$\in$} & \multicolumn{3}{|c|}{$\delta$} \\
\hline & 1 & 0.1 & 0.01 & 1 & 0.1 & 0.01 \\
\hline RPR & $x$ & $\Delta$ & $\mathrm{O}$ & $\mathrm{O}$ & $\Delta$ & $X$ \\
\hline Energy saving & $X$ & $\Delta$ & $\mathrm{O}$ & $\mathrm{O}$ & $\Delta$ & $X$ \\
\hline Throughput & $\mathrm{O}$ & $\Delta$ & $x$ & $x$ & $\Delta$ & $\mathrm{O}$ \\
\hline Initial access & $\mathrm{O}$ & $\Delta$ & $x$ & - & - & - \\
\hline
\end{tabular}

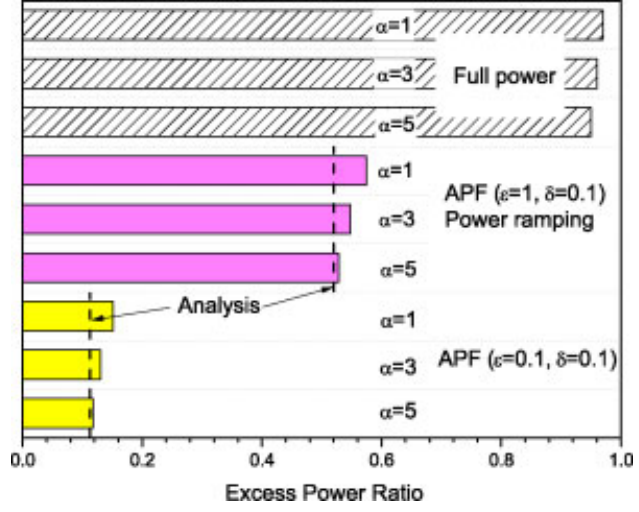

Figure 6. Comparison of the excess-power ratio according to three $\alpha \mathrm{s}(G=0.75$ in steady-state).

Figure 6 shows the EPR of the full-power and APF schemes according to three $\alpha \mathrm{s}$. In any scheme, the EPR increases with the decrease of $\alpha$, since it activates the capture effect more vigorously. As stated earlier, the result of $\alpha=5$ is close to analysis. When $\varepsilon=0.1$ and $\delta=0.1$ in APF, the EPR is very small. When $\varepsilon=1$, the EPR increases and this means that the aggressive increase of transmit power like power ramping causes many users to transmit with excessive power. In the full-power scheme, most users transmit with excessive power inefficiently. We here omit the result of the random-power scheme that shows EPRs in the middle of the two APF schemes.

\subsubsection{Mobile users experiencing fast fading.}

Thus far, we have only considered a static channel environment, where users are affected by path loss and shadowing. Mobile users change their location, and may thus experience fast fading. Using simulation, we show that APF is also applicable to such users. We consider a simple case when users experience i.i.d. Rayleigh fading. To generate Rayleigh fading in the simulator, we use the Jakes model for the mobility of $3 \mathrm{~km} / \mathrm{h}$ [32].

In a fast-fading environment, a random access can be successful at any power level, while there is a reference power level in a static channel environment. Figure 7 depicts the three samples of transmit power levels when Rayleigh fading exists in addition to the average reference level of 5 when $G=0.75$. When $\varepsilon=0.1$ and $\delta=0.01$, APF adapts itself well to the short-term channel condition although it changes slowly. On the other hand, when $\varepsilon=1$ and $\delta=1$, APF tracks the instantaneous channel condition very quickly.
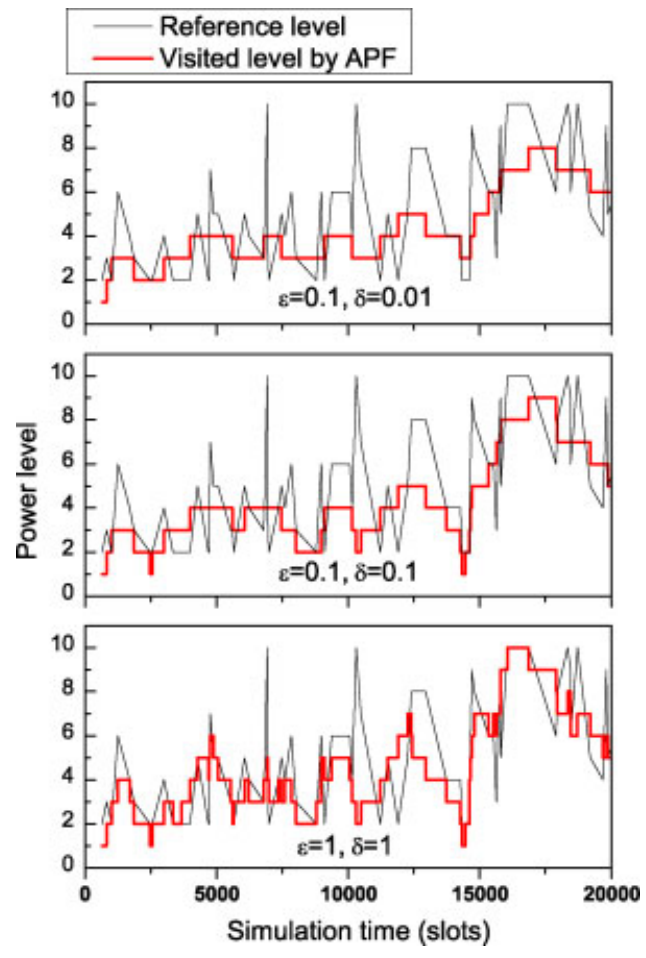

Figure 7. Samples of power-level transitions in a Rayleigh fading environment $(G=0.75)$ obtained from simulation.

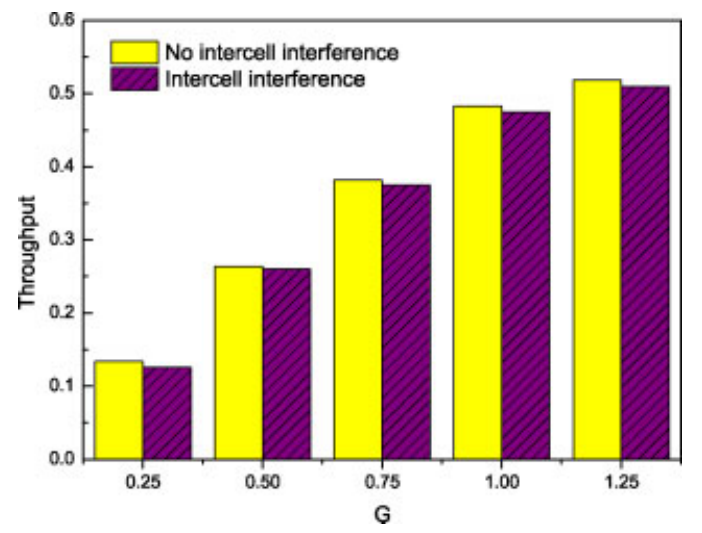

Figure 8. The impact of intercell interference on throughput when the frequency reuse factor of 1 is used $(\varepsilon=0.1, \delta=0.1)$. 


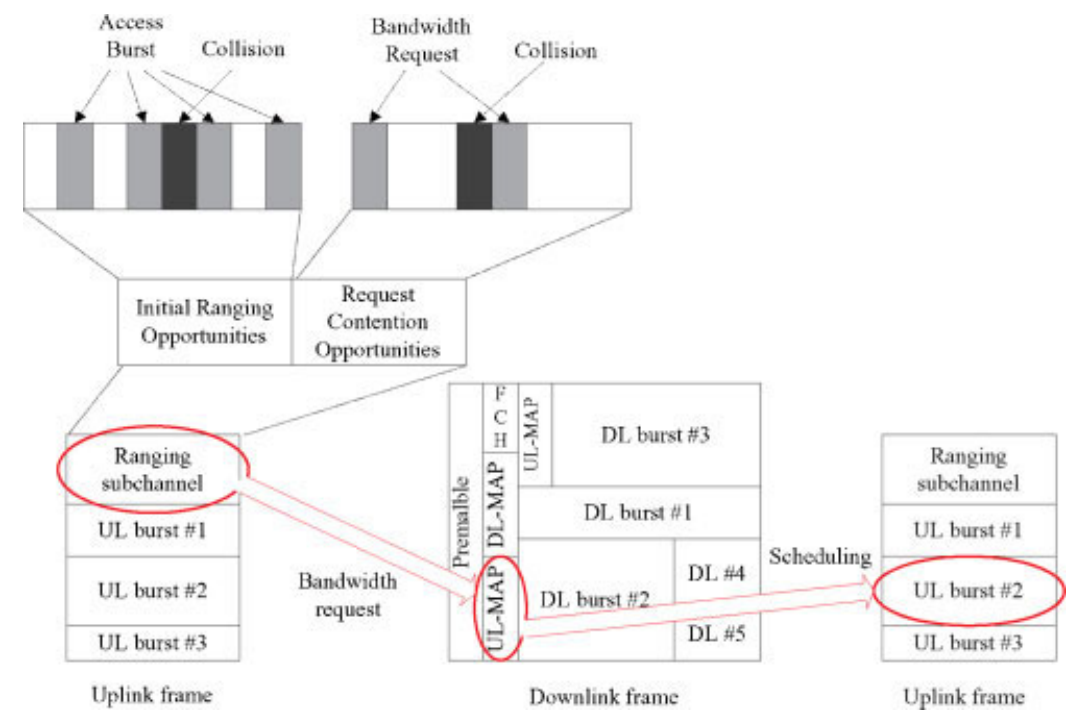

Figure 9. Random access channels defined in the IEEE 802.16 standard.

\subsubsection{Effect of intercell interference.}

In the numerical results presented thus far, we have not considered the effect of intercell interference, i.e., the impact of intercell interference is assumed to be very little for a random access channel. When the frequency reuse of 1 is employed for all cells, some random accesses generated in a cell may be interfered with those in the neighboring cells. We tested its effect by using simulation, when there are six neighboring cells as in the hexagonal cell model. From this simulation, we obtained that about $0.9 \%$ of random accesses generated in neighboring cells caused greater intercell interference than the background noise of $-96 \mathrm{dBm}$, when the neighboring cells are fully loaded (i.e., $G=1$ ). As a result, the throughput and the total consumed energy with the intercell interference accounted for show almost the same as those without considering the intercell interference. Figure 8 shows that the throughput with the intercell interference is slightly less than that without considering it. We omit the comparison of energy consumptions, since they are also very close to each other.

\section{IMPLEMENTATION ISSUE}

As an implementation example, we describe the random access procedure, ranging and bandwidth request, defined in the IEEE 802.16 systems [2]." Since the BS assigns uplink channels to users by uplink scheduling, the users should send their bandwidth needs to the BS. If such a request is successfully received and then honored by the $\mathrm{BS}$, the corresponding bandwidth assignment is broadcast via the uplink MAP (UL-MAP) over a downlink. Then, a

\footnotetext{
\# As mentioned in Section 1, FDD systems (i.e., 802.16-FDD) are closer to our system model than time division duplexing (TDD) systems.
}

user who is assigned a part of the frame will transmit his packet based on the MAP information. A bandwidth request is made for every frame, because uplink scheduling should be performed for every frame.

There are two types of ranging: initial ranging and periodic ranging. Initial ranging is used for initial access, while periodic ranging is used to periodically adjust the uplink parameters, which are also essential for OFDM symbol synchronization. The ranging message includes the information of the used transmit power in order to tune the uplink power level of the subsequent messages, so it is possible that a BS collects the distribution of appropriate transmit power levels, i.e., $\Psi(n)$, within its cell.

The procedure of random access in the 802.16 systems is presented in Figure 9 [2]. However, the design of power control is left as a vendor-specific problem. Our $\mathrm{CoF}$ and $\mathrm{CoS}$ algorithms can be used for the power-adjusted random access for the ranging and bandwidth requests. Bandwidth requests can also utilize the power level tuned by the periodic ranging.

\section{CONCLUSION}

In this paper, we developed a novel method called APF for power-adjusted random access. APF consists of $\mathrm{CoF}$ resolution and $\operatorname{CoS}$ decision, and probabilistically adjusts transmit power for random access networks. The numerical results confirm that APF finely tunes to a proper power level, thus making energy-savings in fixed and mobile wireless environments. It would be interesting to investigate the performance of exponential backoffs combined with APF and its application to multi-hop networks. 


\section{ACKNOWLEDGEMENTS}

This work was supported in part by the MKE (The Ministry of Knowledge Economy) under the ITRC (Information Technology Research Center) support program supervised by the NIPA (National IT Industry Promotion Agency) (NIPA-2010-C1090-1021-0011), World Class University Grant R33-10085, NSF under grants CNS 0519498 and 0721529, as well as Intel Corporation.

\section{REFERENCES}

1. Abramson N. The Aloha system - Another alternative for computer communications. In Proceedings of AFIPS, 1970; 36: pp. 295-298.

2. IEEE 802.16-REVd/D4-2004, Part16: Air Interface for Fixed Broadband Wireless Acces Systems, Mar. 2004.

3. Eklund C, Marks RB, Stanwood KL, Wang S. IEEE standard 802.16: A technical overview of the wirelessMAN air interfance for broadband wireless access. IEEE Communications Magazine 2002; 40(6): 98-107.

4. Choi Y-J, Park S, Bahk S. Multichannel random access in OFDMA wireless networks. IEEE Journal on Selected Areas in Communications 2006; 24(3): 603-613.

5. Fu X, Minn H. Initial uplink synchronization and power control (ranging process) for OFDMA systems. In Proceedings of IEEE Globecom 2004; Dallas, TX, Dec. 2004.

6. Tong L, Naware V. Signal processing in random access. IEEE Signal Processing Magazine 2004; 21(5): 29-39.

7. 3GPP TS 25.321 (V6.2.0), Medium Access Control (MAC) protocol specification (Release 6), Technical Specification Group Radio Access Network, June 2004.

8. Goodman DJ, Saleh AAM. The near/far effect in local Aloha radio communications. IEEE Transactions on Vehicular Technology 1987; VT-36(1): 19-27.

9. Kamerman A, Monteban L. WaveLAN II: a highperformance wireless LAN for the unlicensed band. Bell Labs Technical Journal 1997; 2(3): 118-133.

10. Gobriel S, Melhem R, Mossé D. A unified interference/collision analysis for power-aware adhoc networks. In Proceedings of IEEE INFOCOM 2004, Hong Kong, March 2004.

11. Monks JP, Bharghavan V, Hwu WW. A power controlled multiple access protocol for wireless packet networks. In Proceedings of IEEE INFOCOM 2001; Anchorage, AK, USA, Apr. 2001.

12. Lee CC. Random signal levels for channel access in packet broadcast networks. IEEE Journal on Selected Areas in Communications 1987; 5(6): 1026-1034.

13. LaMaire RO, Krishna A, Zorzi M. On the randomization of transmitter power levels to increase throughput in multiple access radio systems. Wireless Networks 1998; 4(3): 263-277.
14. del Angel G, Fine TL. Optimal power and retransmission control policies for random access systems. IEEE/ACM Transactions on Networking 2004; 12(6): 1156-1166.

15. del Angel G, Fine TL. Information capacity and power control for slotted Aloha random-access systems. IEEE Transactions on Information Theory 2005; 51(12): 4074-4090 .

16. Gao Y, Zeng Z, Kumar PR. Joint random access and power selection for maximal throughput in wireless networks. IEEE INFOCOM 2010; Mar. 2010.

17. Zorzi M, Rao RR. Capture and retransmission control in mobile radio. IEEE Journal on Selected Areas in Communications 1994; 12(8): 1289-1298.

18. Sagduyu YE, Ephremides A. Energy-efficient collision resolution in wireless ad-hoc networks. In Proceedings of IEEE INFOCOM 2003; San Francisco, CA, USA, March 2003.

19. Wu Y, Xia X-G, Zhang Q, Zhu W, Zhang Y-Q. Collision probability and throughput analysis in a powercontrolled DS-CDMA wireless network. IEEE Transactions on Vehicular Technology 2006; 55(1): 350-359.

20. Huang H, Lau VKN. Delay-sensitive distributed power and transmission threshold control for S-ALOHA network with finite state markov fading channels. IEEE Transactions on Wireless Communications 2009; 8(11): 5632-5638.

21. Nie X, Wu C, Chen H. Advanced power calibration for random access in the IEEE 802.16 system. International Conference on Application of Information and Communication Technologies (AICT) 2009; pp. 1-6 Oct. 2009.

22. Ko S, Chang K. Fairness-insured aggressive sub-channel allocation and efficient power allocation algorithms to optimize the capacity of an IEEE 802.16e OFDMA/TDD cellular system. KSII Transactions on Internet and Information Systems 2009; 3(4): 385-398.

23. Yang Y, Yum T-SP. Analysis of power ramping schemes for UTRA-FDD random access channel. IEEE Transactions on Wireless Communication 2005; 4(6): 2688-2693.

24. Qiao D, Choi S, Jain A, Shin K. Miser: An optimal low-energy transmission strategy for IEEE $802.11 \mathrm{a} / \mathrm{h}$. In Proceedings of ACM MobiCom 2003; San Diego, CA, USA, Sept. 2003.

25. Kim T-S, Lim H, Hou JC. Improving spatial reuse through tuning transmit power, carrier sense threshold, and data rate in multihop wireless networks. In Proceedings of ACM MobiCom 2006; Los Angeles, CA, USA, Sept. 2006.

26. Arnbak JC, Blitterswijk WV. Capacity of slotted Aloha in Rayleigh-fading channels. IEEE Journal on Selected Areas in Communication 1987; 5(2): 261-269.

27. Erceg V, Greenstein LJ, Tjandra S, Parkoff SR, Gupta A, Kulic B, Julius A, Jastrzab R. An empirically based 
path loss model for wireless channels in suburban environments. IEEE Journal on Selected Areas in Communication 1999; 17(7): 1205-1211.

28. Dimic G, Sidiropoulos ND, Zhang R. Medium access control - physical cross-layer design. IEEE Signal Processing Magazine 2004; 21(5): 40-50.

29. Kleinrock L. Queueing Systems - Volume 1, Theory, Wiley-Interscience: 1975.

30. Recommmendation ITU-R P.1411-3, "Propagation data and prediction methods for the planning of shortrange outdoor radio communication systems and radio local area networks in the frequency range $300 \mathrm{MHz}$ to 100GHz."

31. Rappaport TS. Wireless Communications: Principles and Practice. Prentice-Hall: Upper Saddle River, NJ, 2001.

32. Jakes WC. Microwave Mobile Communications. John Wiley \& Sons: 1994.

\section{AUTHOR'S BIOGRAPHY}

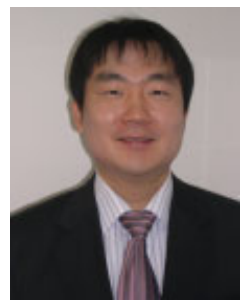

Young-June Choi received B.S., M.S., and $\mathrm{Ph}$.D. degrees from the Department of Electrical Engineering \& Computer Science, Seoul National University, in 2000, 2002, and 2006, respectively. From Sept. 2006 through July 2007, he was a postdoctoral researcher at the University of Michigan, Ann Arbor, MI, USA. From 2007 through 2009, he was with NEC Laboratories America, Princeton, NJ, USA, as research staff member. He is currently an assistant professor at Ajou University, Suwon, Korea. His research interests include fourth-generation wireless networks, radio resource management, and cognitive radio networks. He was the recipient of the Gold Prize at the Samsung Humantech Thesis Contest in 2006.

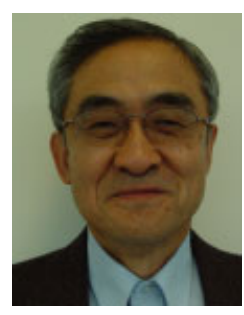

Kang G. Shin is the Kevin and Nancy O'Connor Professor of Computer Science and Founding Director of the Real-Time Computing Laboratory in the Department of Electrical Engineering and Computer Science, The University of Michigan, Ann Arbor, Michigan. His current research focuses on computing systems and networks as well as on embedded real-time and cyber-physical systems, all with emphasis on timeliness, security, and dependability. He has supervised the completion of $67 \mathrm{PhDs}$, authored/coauthored more than 740 technical articles (263 of which are published in archival journals) and more than 20 patents or invention disclosures. He has co-authored (with C. M. Krishna) a textbook "Real-Time Systems," McGraw Hill, 1997. He has received numerous best paper awards, including the Best Paper from the 2010 USENIX Annual Technical Conference, the IEEE Communications
Society William R. Bennett Prize Paper Award in 2003, the Best Paper Award from the IWQoS'03 in 2003, and an Outstanding IEEE Transactions of Automatic Control Paper Award in 1987. He has also coauthored papers with his students which received the Best Student Paper Awards from the 1996 IEEE Real-Time Technology and Application Symposium, and the 2000 UNSENIX Technical Conference. He has also received several institutional awards, including the Research Excellence Award in 1989, Outstanding Achievement Award in 1999, Service Excellence Award in 2000, Distinguished Faculty Achievement Award in 2001, and Stephen Attwood Award in 2004 from The University of Michigan (the highest honor bestowed to Michigan Engineering faculty); a Distinguished Alumni Award of the College of Engineering, Seoul National University in 2002; 2003 IEEE RTC Technical Achievement Award; and 2006 Ho-Am Prize in Engineering (the highest honor bestowed to Korean-origin engineers). He received the B.S. degree in Electronics Engineering from Seoul National University, Seoul, Korea in 1970, and both the M.S. and Ph.D degrees in Electrical Engineering from Cornell University, Ithaca, New York in 1976 and 1978, respectively. From 1978 to 1982 he was on the faculty of Rensselaer Polytechnic Institute, Troy, New York. He also chaired the Computer Science and Engineering Division, EECS Department, The University of Michigan for three years beginning January 1991. He has held visiting positions at the U.S. Airforce Flight Dynamics Laboratory, AT $\& \mathrm{~T}$ Bell Laboratories, Computer Science Division within the Department of Electrical Engineering and Computer Science at UC Berkeley, and International Computer Science Institute, Berkeley, CA, IBM T. J. Watson Research Center, Software Engineering Institute at Carnegie Mellon University, HP Research Laboratories, Hong Kong University of Science and Technology, Ewha Womans University in Korea, and Ecole Polytechnique Federale de Lausanne (EPFL) in Switzerland. He is Fellow of IEEE and ACM, and overseas member of the Korean Academy of Engineering, has chaired numerous major conferences, including 2009 ACM Annual International Conference on Mobile Computing and Networking (MobiCom'09), 2008 IEEE Communications Society Conference on Sensor, Mesh and Ad Hoc Communications and Networks (SECON'08), the 3rd ACM/USENIX International Conference on Mobile Systems, Applications, and Services (MobiSys'05), 2000 IEEE Real-Time Technology and Applications Symposium (RTAS'00), and 1987 IEEE Real-Time Systems Symposium (RTSS). He also chaired the IEEE Technical Committee on Real-Time Systems during 1991-93, was a Distinguished Visitor of the Computer Society of the IEEE, an Editor of IEEE Trans. on Parallel and Distributed Computing, and an Area Editor of International Journal of Time-Critical Computing Systems, Computer Networks, and ACM Transactions on Embedded Systems. He has also served or is serving on numerous government committees, such as the US NSF Cyber-Physical Systems Executive Committee and the Korean Government R \& D Strategy Advisory Committee. 